Original Research Paper

\title{
A Case Vignette and Review of Maladaptive Denial and Related Ethical Issues
}

\author{
${ }^{1}$ Merideth Prevost, ${ }^{2}$ Wendy H. Gerstein, ${ }^{3}$ Natalie M. Salas, ${ }^{4}$ Cynthia Geppert and ${ }^{5}$ Holly Fleming \\ ${ }^{1-3,5}$ Department of Internal Medicine, \\ New Mexico VA Healthcare Systems and University of New Mexico, Albuquerque, New Mexico \\ ${ }^{4}$ Department of Psychiatry, \\ New Mexico VA Healthcare Systems and University of New Mexico, Albuquerque, New Mexico
}

\author{
Article history \\ Received: 16-05-2016 \\ Revised: 08-11-2016 \\ Accepted: 12-11-2016 \\ Corresponding Author: \\ Wendy H. Gerstein \\ Department of Medicine, New \\ Mexico VA Healthcare \\ Systems and University of New \\ Mexico, 1501 San Pedro SE, \\ Albuquerque, New Mexico \\ $\mathrm{Ph}: 505-401-2128$ \\ Fax: 505-256-2803 \\ Email: wendy.gerstein@va.gov
}

\begin{abstract}
Maladaptive denial is a pathological reaction to the symptoms, signs or diagnosis of a physical illness. The dominant response is persistent denial of having the physical disorder. Maladaptive denial typically results in the inability of the patient to provide informed consent to treatment due to the refusal to acknowledge an underlying disease process. This pathologic behavior exposes the affected individual to a significantly higher risk of serious physical illness or death. This paper describes a patient with maladaptive denial to his diagnosis of stage IV Non-Hodgkin's lymphoma which results in his death. The authors de fine the disorder, discuss the common ethical issues that arise when taking care of patients with maladaptive denial and summarize the recommended management.
\end{abstract}

Keywords: Medical Ethics, Surrogate Decision Maker, Maladaptive Denial, Informed Consent

\section{Introduction}

In the summer of 2015, a 58 year old male was admitted to the New Mexico VA Hospital in Albuquerque, NM with stage IV Non-Hodgkin's lymphoma. Four months prior, an outpatient PET CT scan had revealed metabolically active thickened gastric folds and diffuse lymphadenopathy, findings suspicious for malignancy. Initial gastric biopsies were non-diagnostic so endoscopy for repeat biopsies was scheduled. The patient subsequently "no-showed" five appointments and never underwent repeat biopsy. At hospital admission, the patient had profound anasarca, severe hypoalbuminemia and anemia. Imaging revealed right retroperitoneal adenopathy with abscess, interval progression of the lymphadenopathy, inferior vena cava compression and new skeletal lesions. He underwent abscess drainage, treatment, and biopsy. The biopsy was diagnostic for Stage IV Non-Hodgkin's lymphoma. At the time of admission to the inpatient medicine teaching service, the patient was oriented to place and time and could reasonably discuss current events. He appeared to have decisional capacity, he was not delirious and he had no prior history of psychiatric illness.

\section{Need Time to Absorb this Information}

The Oncologist recommended a bone marrow biopsy for staging. The patient declined, stating that he "wanted time to absorb the information." $\mathrm{He}$ also made statements such as "I know my body better than you," and "I need to get my paperwork in order before having the procedure." The bone marrow biopsy was performed six days later due to patient delays.

\section{Just Need to Eat Better and My Blood Counts Will Go $U p$}

A blood transfusion was recommended prior to initiation of chemotherapy. The patient refused, stating that he would only accept his own blood and "I just need to eat better and my blood counts will go up." He became fixated on completing an advanced directive. The patient named his sister as a healthcare surrogate, but he forbid the medical team from contacting her regarding his health status. He wanted full resuscitative efforts under all clinical scenarios and specified that these preferences be followed strictly.

\section{You are Just Negative People}

The Psychiatry team was consulted due to the patients' evolving patterns of denial which resulted in refusal of interventions and caused treatment delays. The psychiatrists had concerns about the patient's ability to understand his disease and his ability to navigate complex medical decisions. They felt the patient was exhibiting a fixed belief system based on his statements that "God has a plan for me," "God uses doctors to heal 
people" and "I know that I am going to survive this."The patient was unwilling to participate in discussions about the possibility of death. When providers attempted to discuss his critical illness, he called them "negative people." When code status was discussed the patient stated "Of course, I want everything done."Chemotherapy informed consent discussions were not possible because the patient was unwilling to acknowledge that he had cancer and would not discuss the potential adverse outcomes of treatment. Nevertheless, he remained adamant about wanting to proceed with chemotherapy.

Several weeks into his hospitalization the patient began to clinically decompensate with intermittent periods of delirium. The Psychiatry team determined he no longer had decisional capacity and the medicine team asked his sister to act as his surrogate using a shared decision-making model.

\section{Think I Need a Vacation from Albuquerque}

The providers counseled the sister on the risks and benefits of proceeding with chemotherapy. She confirmed that the patient "had always been in denial" when it came to medical matters. She discussed with the patient his diagnosis, treatment options and the high likelihood of a poor outcome regardless of whether or not he underwent chemotherapy. Using substituted judgment, she decided that he would want to proceed with chemotherapy. Within three days of initiating chemotherapy the patient developed acute renal and respiratory failure. The sister felt that she had fulfilled the patient's wishes for aggressive treatment and due to his further clinical deterioration, she requested that he be DNR/DNI and wanted aggressive treatment discontinued. The patient was placed on comfort measures and died after 54 days in the hospital. Just prior to his death, the patient stated, "I think I need a vacation from Albuquerque."

\section{Maladaptive Denial}

The term denial is used extensively in the psychiatric and medical literature. Denial is defined as a refusal to admit the truth or reality, or a psychological defense mechanism in which confrontation with a personal problem is avoided by denying the existence of the problem (Muskin et al., 1998). There is debate regarding the best way to define denial and there is recognition in the literature that denial can be either adaptive or maladaptive. A certain level of denial can be helpful, allowing patients to cope with a serious diagnosis (Strauss et al., 1990; Vos et al., 2011). As denial becomes more severe, it becomes a hindrance to the patient's ability to cope with their diagnosis and seek appropriate treatment. Severe denial can even cause the patient to lack the capacity to perceive facts regarding a specific medical diagnosis (Russell, 1993; Shelp and Perl, 1985).
The accepted definition of maladaptive denial is a reaction to the symptoms, signs or diagnosis of a physical illness, with the predominant response being persistent denial of having that physical illness. The affected individual asserts that he or she does not have the physical disease or behaves in a way that indicates that he or she minimizes the significance of the disease. This type of denial occurs in the face of obvious physical manifestations of the disease, or in spite of the patient being informed of its presence by doctors. This type of denial exposes the individual to a significantly higher risk of serious physical illness or death (Muskin et al., 1998).

The following conditions and circumstances preclude a patient from being described as having maladaptive denial:

- Patients with known psychotic disorders

- Patients who refuse treatment who have decisional capacity and who have participated in the informed consent process

- Patients who refuse treatment because it violates their religious beliefs or subcultures

Several authors discuss the dangers of using the term denial as it often represents conflicts between patients and clinicians rather than a true diagnostic entity. Denial should not be used to describe those who simply disagree with their physician's opinions because this does not typically represent being out of touch with reality. Nevertheless, patients are often labeled as "noncompliant" and "deniers" when they just have different belief systems. These negative categorizations can cause physicians to disregard the wishes of the patient, leading to a decrease in autonomy for the patient. There can also be an increased use of paternalism when patients are mislabeled as denying. Only in cases of true maladaptive denial with loss of decisional capacity is it justifiable to use a paternalistic approach, which limits the patient's autonomy (Shelp and Perl, 1985).

This patient met the current criteria for maladaptive denial that is described in the literature (Kunkel et al., 1997). The earliest manifestation of this disorder was his non-compliance with outpatient appointments resulting in failure to timely diagnose his cancer. His late presentation to the hospital with severe disease was also consistent with maladaptive denial. Despite numerous attempts by physicians to have informed consent discussions with the patient, he repeatedly created alternative explanations for his symptoms. He insisted that his prior reality (i.e., his healthy self) still held true and refused to acknowledge his diagnosis. The patient had no history of a psychiatric illness or dementia and was not delirious at admission to the hospital. He had no history of substance abuse and did not ascribe to any religious belief that would preclude treatment. 
Table 1 . The components of decisional capacity and requirements for an informed consent discussion

Decisional capacity assessment:

The patient is able to:

- Communicate preferences.

- Comprehend the gravity of her/his situation and decision.

- Reason through/manipulate information.

- Make authentic choices.

Provisions for an informed consent discussion include:

- A clear diagnosis and prognosis is explained to the patient.

- An explanation of the nature of the intervention.

- An explanation of the risks of the intervention.

- An explanation of the benefits of the intervention.

- Alternatives to the intervention, their risks and benefits, including no intervention.

\section{Ethical Issues}

There are three important ethical questions raised in this case:

1) Cana patient diagnosed with maladaptive denial possess decisional capacity and participate in an informed consent discussion?

Maladaptive denial does not automatically confer decisional incapacity across all decision making domains. Decisional capacity should be viewed along a spectrum, with each type of decision requiring a separate assessment of capacity (Ganzini et al., 2005). The components of decisional capacity and requirements for an informed consent discussion are listed above in Table 1 (Appelbaum, 2007).

Decisional capacity is a prerequisite for an informed consent discussion. Our patient demonstrated decisional capacity in all other aspects of his life except issues related to his physical illness. He was oriented to place, date, time and was able to discuss current events and the need to manage his finances. His persistent inability to acknowledge his diagnosis made it impossible for physicians to discuss with him the risks and benefits of treatment options, or alternatives to aggressive treatment. Because of this maladaptive denial, the patient lacked decisional capacity to provide informed consent for treatment:

2) Was it ethical to disregard the patient's advanced directives?

The patient's living will requested that the provision of life-sustaining treatment be administered in all clinical scenarios and he specified that his surrogate strictly follow his preferences. The Ethics consultants identified that his directive was completed at the height of the patient's maladaptive denial and therefore, was invalid due to his decisional incapacity related to healthcare decisions. Ethically his surrogate had the authority and responsibility to make medical decisions using substituted judgment.

3) Given that the patient lacked decisional capacity upon execution of his advanced directives, was it ethically justifiable to use his sister as a surrogate?

The surrogate was contacted over the objections of the patient because it was believed that there was a higher obligation to avoid doing harm than the obligation to maintain autonomy and confidentiality. Although the patient lacked decisional capacity for healthcare decisions, he did have the capacity to recognize and choose his surrogate decision maker. An individual acting on his behalf and in accordance with his core wishes and values was necessary to ensure his authentic preferences were honored. The patient identified his sister as his surrogate because he believed she would act in his best interest. His sister was familiar with the patient's mindset, enabling her to use substituted judgement for medical decisions. In addition, his sister was his only known living relative, making her the appropriate legal surrogate.

\section{Management Strategies}

Early recognition of maladaptive denial could have been life-saving for this patient. Providers should be alarmed when a patient is informed that they have a possible malignancy and subsequently miss all of their outpatient follow-up appointments. This behavior pattern, in conjunction with a late presentation of disease, should trigger providers to obtain early psychiatric intervention. The initial medical and psychiatric evaluations should include an assessment of decisional capacity, specifically the capacity for healthcare decisions. 
Surrogate identification is critical for patients with maladaptive denial. Although it is important to allow a patient to participate in the decision process in an effort to try to understand their values and uphold their autonomy, a surrogate is needed to provide informed consent. In this case, the surrogate became involved quite late and during a time of significant stress. The providers were faced with a patient who was terminally ill and clinically deteriorating, yet demanding full resuscitative efforts. Earlier surrogate involvement may have alleviated some of the uncertainty and delays that were associated with treatment decisions in this patient who was critically ill, yet unable to consent.

Gold beck counsels that maladaptive denial is best tackled with a non-judgmental stance (Goldbeck 1997). A confrontational approach will often result in increased denial, decrease in treatment compliance and can precipitate a complete breakdown of the doctor-patient relationship. Psychiatry experts should be consulted in order to model and provide this non-confrontational approach.

Minimizing the number of provider-patient encounters may have improved the patient's quality of life during this hospitalization. Although a multidisciplinary approach is indicated for patients with an active malignancy and complex psychiatric issues, the volume of teams interacting with this patient resulted in significant confusion, distrust and emotional distress for this patient. It is recommended to identify a primary medical team as the point of contact for patient care in patients with maladaptive denial, which can decrease the stress for the patient and minimize miscommunication between providers.

\section{Conclusion}

Maladaptive denial can be difficult to initially recognize. Multiple missed appointments and an inability to acknowledge a disease are important warning signs in a patient who has been recently diagnosed with a serious illness. Providers need to be aware that maladaptive denial can compromise a patient's decisional capacity and their ability to provide informed consent. Early psychiatric intervention, establishing a surrogate, and a employing non-confrontational approach may be life-saving for these patients.

\section{Acknowledgement}

No financial support provided.

\section{Author's Contributions}

Each author individually contributed to the preparation, development and publication of this manuscript.

\section{Ethics}

There are currently no known ethical issues that could arise from publication of this manuscript.

\section{References}

Muskin, P.R., T. Feldhammer, J.L. Gelfand and D.H. Strauss, 1998. Maladaptive denial of physical illness: A useful new "diagnosis". Int. J. Psychiatry Med., 28: 463-477.

DOI: 10.2190/0KWM-7WJ1-NXNV-W7UQ

Strauss, D.H., R.L. Spitzer and P.R. Muskin, 1990. Maladaptive denial of physical illness: A proposal for DSM-IV. Am. J. Psychiatry, 147: 1168-1172. DOI: 10.1176/ajp.147.9.1168

Vos, M.S., H. Putter, H.C.V. Houwelingen and H.C.J.M. de Haes, 2011. Denial and social and emotional outcomes in lung cancer patients: The protective effect of denial. J. Lung Can., 72: 119-124. DOI: 10.1016/j.lungcan.2010.07.007

Russell, G.C., 1993. The role of denial in clinical practice. J. Adv. Nurs., 18: 938-940. DOI: 10.1046/j.1365-2648.1993.18060938.x

Shelp, E.E. and M. Perl, 1985. Denial in clinical medicine: A reexamination of the concept and its significance. Arch Intern. Med., 145: 697-699. DOI: $10.1001 /$ archinte.1985.00360040129027

Kunkel, E.J.S., C.M. Woods, C. Rodgers and R.E. Myers, 1997. Consultations for 'maladaptive denial of illness' in patients with cancer: Psychiatric disorders that result in noncompliance. PsychoOncology, 6: 139-149. DOI: 10.1002/(SICI)10991611(199706)6:2<139::AID-PON256>3.0.CO;2-O

Ganzini, L., L. Volicer, W.A. Nelson, E. Fox and A.R. Derse, 2005. Ten myths about decision-making capacity. J. Am. Med. Dir. Assoc., 6: S100-S104. DOI: $10.1016 /$ j.jamda.2005.03.040

Appelbaum, P.S., 2007. Assessment of patients' competence to consent to treatment. N Engl. J. Med., 357: 1834-1840. DOI: $10.1056 /$ NEJMcp074045

Goldbeck, R., 1997. Denial in physical illness. J. Psychosomatic Res., 43: 575-593. DOI: $10.1016 / \mathrm{S} 0022-3999(97) 00168-2$ 